\title{
The structure of dissipative viscous system of conservation laws
}

\author{
Denis Serre \\ École Normale Supérieure \\ de Lyon*
}

In honor to Professor Aldo Belleni MoRAnte, on his 70th birthday

\begin{abstract}
We revisit the structure of viscous systems of conservation laws that are entropydissipative. We prove that the dissipated quantities are spatial derivatives of certain non-linear coordinates that are defined only in terms of the entropy and of the linear, non-dissipated, coordinates.
\end{abstract}

Keywords: Systems of conservation laws; dissipative structure; entropy.

We begin with a first-order system of conservation laws

$$
\partial_{t} u+\operatorname{div} f(u)=0, \quad\left(x \in \mathbb{R}^{d}, u(x, t) \in \mathcal{U}\right),
$$

where $\mathcal{U}$ is a convex, open subset of $\mathbb{R}^{n}$. We assume that (1) admits a strongly convex entropy $\eta$ of flux $Q$. Strong convexity just means that $\mathrm{D}^{2} \eta(u)$ is positive definite for every state $u \in \mathcal{U}$.

Let $\bar{u} \in \mathcal{U}$ be given. Without loss of generality, we may assume that $\eta$ reaches its minimum at $\bar{u}$, up to the replacement of $\eta$ by

$$
\hat{\eta}(u):=\eta(u)-\eta(\bar{u})-\mathrm{d} \eta(\bar{u})(u-\bar{u}) .
$$

This assumption allows us to make a priori estimates of weak solutions satisfying the entropy inequality

$$
\partial_{t} u+\operatorname{div} Q(u) \leq 0,
$$

such that $u(\cdot, t)-\bar{u}$ is square integrable.

The purpose of this paper is to study viscous extensions of (1) that are compatible with the entropy, in the sense that there is an entropy inequality for classical solutions of the extended system. In our weakest setting, such an inequality stands at the level of spatial integrals only. Somehow, we justify requirements made by Kawashima in his thesis [9], using only natural assumptions.

*UMPA (UMR 5669 CNRS), ENS de Lyon, 46, allée d'Italie, F-69364 Lyon, cedex 07, FRANCE. 


\section{What is viscous dissipation}

We consider a viscous extension of (1), which has a similar unknown $u$ :

$$
\partial_{t} u+\operatorname{div} f(u)=\operatorname{div}(B(u) \nabla u)=\sum_{\alpha, \beta} \partial_{\alpha}\left(B^{\alpha \beta}(u) \partial_{\beta} u\right) .
$$

Multiplication of $(2)$ by $\mathrm{d} \eta(u)$ yields

$$
\begin{aligned}
\partial_{t} \eta(u)+\operatorname{div} Q(u) & =\mathrm{d} \eta(u) \operatorname{div}(B(u) \nabla u) \\
& =\operatorname{div}(\mathrm{d} \eta(u) B(u) \nabla u)-\sum_{\alpha, \beta} \mathrm{D}^{2} \eta(u)\left(\partial_{\alpha} u, B^{\alpha \beta}(u) \partial_{\beta} u\right),
\end{aligned}
$$

where we treat $\mathrm{D}^{2} \eta(u)$ as a symmetric bilinear form. The last term is quadratic in the Jacobian matrix $\nabla u$. We recall that if $d=n=1$, this term is simply $-b(u) \eta^{\prime \prime}(u)\left(\partial_{x} u\right)^{2}$ and is non-positive because $\eta$ is convex and $b>0$ (for the Cauchy problem to be well-posed for (2).)

At first glance, we might say that the viscous model is weakly entropy-dissipative if (2) implies the inequality

$$
\partial_{t} \eta(u)+\operatorname{div} Q(u) \leq \operatorname{div}(\mathrm{d} \eta(u) B(u) \nabla u),
$$

but this definition is a bit too weak for our concern. Such a system could even be entropyconservative. This is why the following stronger definition has often be prefered (see for instance $[2,9]$ :

Definition 1.1 We say that the viscous model is strongly entropy-dissipative if (2) formally implies an inequality

$$
\partial_{t} \eta(u)+\operatorname{div} Q(u)+\omega \sum_{\alpha}\left|\sum_{\beta} B^{\alpha \beta}(u) \partial_{\beta} u\right|^{2} \leq \operatorname{div}(\mathrm{d} \eta(u) B(u) \nabla u),
$$

where $\omega=\omega(u)$ is strictly positive and continuous. This amounts to saying that

$$
\begin{aligned}
\sum_{\alpha, \beta} \mathrm{D}^{2} \eta(u)\left(X_{\alpha}, B^{\alpha \beta}(u) X_{\beta}\right) \geq & \omega \sum_{\alpha}\left|\sum_{\beta} B^{\alpha \beta}(u) X_{\beta}\right|^{2} \\
& =: \omega|B(u) \mathbf{X}|^{2}, \quad \forall u \in \mathcal{U}, \forall X_{1}, \ldots, X_{d} \in \mathbb{R}^{n} .
\end{aligned}
$$

Note that the term $|B(u) \mathbf{X}|^{2}$ (the reader is warned that the notation (5) is a little bit confusing) is the strongest quadratic form that we may expect to control from the left-hand side of (4), since if it vanishes, then this left-hand side vanishes too.

Definition 1.1 is however a bit too strong, for the following reason. Assume for the moment that the system (2) be linear, with constant coefficients. In particular, $\eta$ is quadratic: $\eta(u)=$ $\frac{1}{2} u^{T} S u$ with $S \in \mathbf{S P D}_{n}$. What we really wish is that

$$
\int_{\mathbb{R}^{d}} \sum_{\alpha, \beta}\left(S \partial_{\alpha} U \mid B^{\alpha \beta} \partial_{\beta} U\right) d x \geq \omega \int_{\mathbb{R}^{d}} \sum_{\alpha}\left|\sum_{\beta} B^{\alpha \beta} \partial_{\beta} U\right|^{2} d x
$$


for every smooth, compactly supported, field $U$. Although (6) is ensured by (4), we do not really need the latter. What we need is only (apply Parseval Formula):

$$
(S X \mid B(\xi) X) \geq \omega \sum_{\alpha}\left|B^{\alpha}(\xi) X\right|^{2}, \quad \forall X \in \mathbb{R}^{n}, \forall \xi \in \mathbb{R}^{d}
$$

where we have defined the partial and total symbols

$$
B^{\alpha}(\xi):=\sum_{\beta} \xi_{\beta} B^{\alpha \beta}, \quad B(\xi)=\sum_{\alpha} \xi_{\alpha} B^{\alpha}(\xi):=\sum_{\alpha, \beta} \xi_{\alpha} \xi_{\beta} B^{\alpha \beta}, \quad\left(\xi \in \mathbb{R}^{d}\right) .
$$

At the quasilinear level, we thus have the

Definition 1.2 We say that the viscous model (2) is entropy-dissipative if

$$
\mathrm{D}^{2} \eta(u)(X, B(\xi ; u) X) \geq \omega(u) \sum_{\alpha}\left|B^{\alpha}(\xi ; u) X\right|^{2}, \quad \forall u \in \mathcal{U}, \forall \xi \in \mathbb{R}^{d}, \forall X \in \mathbb{R}^{n},
$$

where we now take in account the u-dependence of the symbols, and where $u \mapsto \omega(u)$ is still continuous and positive.

We notice that this definition might not be accurate enough, in that we wish to have

$$
\int_{\mathbb{R}^{d}} \sum_{\alpha, \beta} \mathrm{D}^{2} \eta(U)\left(\partial_{\alpha} U, B^{\alpha \beta}(U) \partial_{\beta} U\right) d x \geq \omega_{0} \int_{\mathbb{R}^{d}} \sum_{\alpha}\left|\sum_{\beta} B^{\alpha \beta}(U) \partial_{\beta} U\right|^{2} d x
$$

and this might require a special dependence of $B$ as a function of $u$, while Definition 1.2 affords only for the $\xi$-dependence. However we shall be able to derive interesting results from (8) alone.

\subsection{The vanishing viscosity limit}

To see that (9) is relevant, let us consider the vanishing viscosity limit. We replace $B$ by $\epsilon B$ $(\epsilon>0$ a viscosity parameter) in (2):

$$
\partial_{t} u^{\epsilon}+\operatorname{div} f\left(u^{\epsilon}\right)=\epsilon \operatorname{div}\left(B\left(u^{\epsilon}\right) \nabla u^{\epsilon}\right)=\epsilon \sum_{\alpha, \beta} \partial_{\alpha}\left(B^{\alpha \beta}\left(u^{\epsilon}\right) \partial_{\beta} u^{\epsilon}\right)
$$

We thus have, after multiplication by $\mathrm{d} \eta\left(u^{\epsilon}\right)$,

$$
\partial_{t} \eta\left(u^{\epsilon}\right)+\operatorname{div} Q\left(u^{\epsilon}\right)=\epsilon \operatorname{div}\left(\mathrm{d} \eta\left(u^{\epsilon}\right) B\left(u^{\epsilon}\right) \nabla u^{\epsilon}\right)-\epsilon \sum_{\alpha \beta} \mathrm{D}^{2} \eta\left(u^{\epsilon}\right)\left(\partial_{\alpha} u^{\epsilon}, B^{\alpha \beta}\left(u^{\epsilon}\right) \partial_{\beta} u^{\epsilon}\right)
$$

Let us assume that $\nabla u^{\epsilon}$ and $u^{\epsilon}-\bar{u}$ decay fast enough as $|x| \rightarrow+\infty$, an integration yields

$$
\frac{d}{d t} \int_{\mathbb{R}^{d}} \eta\left(u^{\epsilon}\right) d x+\epsilon \int_{\mathbb{R}^{d}} \sum_{\alpha, \beta} \mathrm{D}^{2} \eta\left(u^{\epsilon}\right)\left(\partial_{\alpha} u^{\epsilon}, B^{\alpha \beta}\left(u^{\epsilon}\right) \partial_{\beta} u^{\epsilon}\right) d x \leq 0 .
$$


Integrating again with respect to time, and using (9), we find

$$
\begin{aligned}
\sup _{t \geq 0} \int_{\mathbb{R}^{d}} \eta\left(u^{\epsilon}(x, t)\right) d x & \leq \int_{\mathbb{R}^{d}} \eta(u(x, 0)) d x, \\
\epsilon \omega_{0} \int_{0}^{T} d t \int_{\mathbb{R}^{d}} \sum_{\alpha}\left|\sum_{\beta} B^{\alpha \beta}\left(u^{\epsilon}\right) \partial_{\beta} u^{\epsilon}\right|^{2} d x & \leq \int_{\mathbb{R}^{d}} \eta(u(x, 0)) d x .
\end{aligned}
$$

From these estimates, it follows that $u^{\epsilon}-\bar{u}$ is bounded in $L_{t}^{\infty}\left(L_{x}^{2}\right)$, while $\epsilon^{1 / 2} B\left(u^{\epsilon}\right) \nabla u^{\epsilon}$ is bounded in $L_{x, t}^{2}$, independently of $\epsilon$. The latter point is extremely useful, since it implies that $\epsilon B\left(u^{\epsilon}\right) \nabla u^{\epsilon}$ tends to zero in $L_{x, t}^{2}$ as $\epsilon \rightarrow 0^{+}$, and therefore the viscous term

$$
\epsilon \operatorname{div}\left(B\left(u^{\epsilon}\right) \nabla u^{\epsilon}\right)
$$

tends to zero in the distributional sense. Thus if in addition we are able to pass to the limit in the nonlinear flux $f\left(u^{\epsilon}\right)$ (for instance if $u^{\epsilon}$ converges almost everywhere ${ }^{1}$ ), then we find that the limit of $u^{\epsilon}$ is the solution of (1).

\section{$1.2 \quad$ Range and kernels.}

Proposition 1.1 Let the viscous system be entropy-dissipative, in the sense of (8). Then

1. one has

$$
\operatorname{ker} B(\xi ; u)=\bigcap_{\alpha} \operatorname{ker} B^{\alpha}(\xi ; u), \quad \forall \xi \in \mathbb{R}^{d},
$$

2. the spectrum of the symbol $B(\xi ; u)$ is contained in the union of the right half-plane $\{z ; \operatorname{Re} z>0\}$ and of the origin $z=0$,

3. the kernel of $B(\xi ; u)$ and its range are orthogonal with respect to the scalar product defined by $\mathrm{D}^{2} \eta(u)$,

4. the zero eigenvalue is semi-simple (that is, its multiplicity equals the dimension of the kernel).

\section{Proof}

- The first point follows immediately from (8) and the definition of the symbols.

\footnotetext{
${ }^{1}$ The convergence almost everywhere is of course a very difficult, largely open, issue. It is the question of stability, in some appropriate topology where derivatives are under control. What we have discussed so far is merely the consistency of the vanishing viscosity method. Consistency is the fact that if the approximate solution converges to something, then the limit is a solution of the limit problem.
} 
- Decomposing a vector into its real and imaginary parts, we see that we also have

$$
\operatorname{Re}^{2} \eta(u)(\bar{X}, B(\xi ; u) X) \geq \omega \sum_{\alpha}\left|B^{\alpha}(\xi ; u) X\right|^{2}, \quad \forall X \in \mathbb{C}^{n} .
$$

When $X$ is an eigenvector of $B(\xi ; u)$, with $\lambda$ the eigenvalue, there comes

$$
(\operatorname{Re} \lambda) \mathrm{D}^{2} \eta(u)(\bar{X}, X) \geq \omega \sum_{\alpha}\left|B^{\alpha}(\xi ; u) X\right|^{2} \geq 0
$$

Since $\mathrm{D}^{2} \eta>0_{n}$, the factor $\mathrm{D}^{2} \eta(u)(\bar{X}, X)$ is positive. There follows $\operatorname{Re} \lambda \geq 0$, with equality only if $B^{\alpha}(\xi ; u) X=0$ for every $\alpha$, which implies $B(\xi ; u) X=0$. In this latter case, we have $\lambda=0$.

- Let $Y$ belong to the kernel, and $X$ be an arbitrary vector. Apply the dissipation (8) to $X+s Y$. Since we also have $B^{\alpha}(\xi ; u) Y=0$ (see the previous point), there remains

$$
\mathrm{D}^{2} \eta(u)(s Y+X, B(\xi ; u) X) \geq \omega \sum_{\alpha}\left|B^{\alpha}(\xi ; u) X\right|^{2}
$$

Since $s \in \mathbb{R}$ is arbitrary, this implies

$$
\mathrm{D}^{2} \eta(u)(Y, B(\xi ; u) X)=0
$$

This is the orthogonality of the kernel and the range of $B(\xi ; u)$, with respect to the scalar product induced by $\mathrm{D}^{2} \eta(u)$.

- The orthogonality, plus the fact that $\mathrm{D}^{2} \eta(u)$ is positive definite, imply that the intersection of the kernel and the range is trivial. Because of dimensionality, this means

$$
\mathbb{R}^{n}=\operatorname{ker} B(\xi, u) \oplus R(B(\xi ; u))
$$

This exactly tells us that 0 is semi-simple.

In particular, the knowledge of either the kernel or the range determines completely the other; if one of both is independent of $\xi \neq 0$, the other one is so, too. Since in practice, the range of $B(\xi ; u)$ does not depend on $\xi$ (Assumption $\mathbf{A}$ below), we deduce that the kernel does not as well. The latter fact was one of Kawashima's assumptions in his thesis [9]. This fact is illustrated by the Navier-Stokes system, where the kernel has dimension one (it is the tangent space to the line $\{v=$ cst, $\theta=$ cst $\}$ ) and the null form $\ell$ is obviously constant, corresponding to the conservation of mass. In general, the kernel does depend on $u$ (though not upon $\xi$, because of the proposition above), unlike the range. 


\section{The nature of dissipated quantities}

Physically relevant viscous models not only have a dissipative structure. They also contain a few first-order conservation laws. This means that there exist linear forms (i.e. coordinates) $\ell$, such that $\ell B(\xi ; u) \equiv 0$ for every state $u$ and frequency $\xi$. With a linear change of coordinates, we may always assume that the $p$ first rows of $B(\xi ; u)$ are null, so that the system contains the conservation laws ${ }^{2}$

$$
\partial_{t} u_{j}+\sum_{\alpha} \partial_{\alpha} f_{j}^{\alpha}(u)=0, \quad j=1, \ldots p
$$

A typical illustration is of course the conservation of mass in continuum mechanics, which in the Navier-Stokes system remains the same as in the Euler system:

$$
\partial_{t} \rho+\operatorname{div}(\rho v)=0 \quad \text { (no right-hand side here; just zero.) }
$$

Another one is the conservation of momentum in gas dynamics, if we neglect the Newtonian viscosity and keep only the thermal diffusion (Fourier-Euler system).

We notice that (13) implies that the ranges of each $B^{\alpha \beta}(u)$, and therefore that of $B(\xi ; u)$, are included in the fixed subspace $\{0\} \times \mathbb{R}^{n-p}$. In the sequel, we make the rather natural

Assumption A: for every $\xi \neq 0$, the rank of the symbol $B(\xi ; u)$ is precisely $n-p$.

In other words, the range $R(B(\xi ; u))$ is not only contained in, but is equal to $\{0\} \times \mathbb{R}^{n-p}$, for every non-zero frequency $\xi$. It amounts to saying that $B(\xi ; u)$ has a constant range for $\xi \neq 0$. We point out that we do not need to consider each $B^{\alpha}(\xi ; u)$ separately.

Remark. Amazingly, we do not need to assume the Onsager relations (micro-reversibility) $B^{\alpha \beta}(u)=B^{\beta \alpha}(u)^{T}$.

Recall now Proposition 1.1: ker $B(\xi ; u)$ is $\mathrm{D}^{2} \eta(u)$-orthogonal to $R(B(\xi ; u))$. In other words, when $\xi \neq 0$, ker $B(\xi ; u)$ is defined by the following linear equations

$$
\operatorname{ker} B(\xi ; u)=\left\{z \in \mathbb{R}^{n} ;\left(\mathrm{d} \frac{\partial \eta}{\partial u_{j}}\right) z=0, \forall j \geq p+1\right\} .
$$

Because of (12), we deduce that the kernel of $B^{\alpha}(\xi ; u)$ contains

$$
\bigcap_{j=p+1}^{n} \operatorname{ker} d \frac{\partial \eta}{\partial u_{j}}, .
$$

Let $\left\{\mathbf{v}_{1}, \ldots, \mathbf{v}_{n}\right\}$ be the dual basis of

$$
\left\{\mathrm{d} \frac{\partial \eta}{\partial u_{1}}, \ldots, \mathrm{d} \frac{\partial \eta}{\partial u_{n}}\right\} .
$$

\footnotetext{
${ }^{2}$ Of course, system (13) is not closed, since the fluxes also involve the components $u_{p+1}, \ldots, u_{n}$.
} 
It is formed of the columns of the matrix $\mathrm{D}^{2} \eta^{*}$, with $\eta^{*}$ the Legendre transform of $\eta$. In particular, it depends smoothly on $u$. Denoting

$$
Y_{j}^{\alpha}(\xi ; u):=B^{\alpha}(\xi ; u) \mathbf{v}_{j}
$$

so that $Y_{1}^{\alpha}=\cdots=Y_{p}^{\alpha}=0$, we can write

$$
B^{\alpha}(\xi ; u)=\sum_{j=1}^{n} Y_{j}^{\alpha} \mathrm{d} \frac{\partial \eta}{\partial u_{j}}=\sum_{j=p+1}^{n} Y_{j}^{\alpha} \mathrm{d} \frac{\partial \eta}{\partial u_{j}}
$$

Remarking that

$$
Y_{j}^{\alpha}(\xi ; u)=\sum_{\beta=1}^{d} \xi_{\beta} Y_{j}^{\alpha \beta}(u), \quad Y_{j}^{\alpha \beta}:=B^{\alpha \beta} \mathbf{v}_{j}
$$

we find

$$
B^{\alpha \beta}(u)=\sum_{j=p+1}^{n} Y_{j}^{\alpha \beta} \mathrm{d} \frac{\partial \eta}{\partial u_{j}} .
$$

Our first conclusion is that the fluxes $B(u) \nabla u$ in the second-order terms involve only the first order derivatives of

$$
\frac{\partial \eta}{\partial u_{p+1}}, \ldots, \frac{\partial \eta}{\partial u_{n}}
$$

namely:

$$
B^{\alpha \beta} \partial_{\beta} u=\sum_{j=p+1}^{n} Y_{j}^{\alpha \beta} \partial_{\beta} \frac{\partial \eta}{\partial u_{j}} .
$$

We now establish a property of the vectors $Y_{j}^{\alpha \beta}(u)$. To begin with, they belong to $R\left(B^{\alpha \beta}(u)\right)$ and therefore their coordinates $Y_{j k}^{\alpha \beta}(u)$ vanish for $k \leq p$. Let us next rewrite (8) in terms of the tensor $Y$. We shall employ as usual the symbols $Y^{\alpha}(\xi ; u)$ and $Y(\xi ; u)$, which are $n \times n$ matrices. Their columns are $Y_{j}^{\alpha}$ and $Y_{j}$, respectively, while their entries are $Y_{i j}^{\alpha}$ and $Y_{i j}$. We point out that, since $Y_{j}^{\alpha \beta}$ belongs to the range of $B^{\alpha \beta}$, these entries vanish for $i=1, \ldots, p$ : we may write

$$
Y_{j}^{\alpha \beta}=\left(\begin{array}{c}
0 \\
Z_{j}^{\alpha \beta}
\end{array}\right) .
$$

Since the rank of $B(\xi)$ equals $n-p$, the rank of $\left\{Z_{p+1}(\xi), \ldots, Z_{n}(\xi)\right\}$ equals $n-p$ when $\xi \neq 0$.

Making the linear transformation

$$
X \mapsto V:=\mathrm{D}^{2} \eta(u) X,
$$

we have

$$
B^{\alpha}(\xi ; u) X=\sum_{j \geq p+1} v_{j} Y_{j}^{\alpha}(\xi), \quad B(\xi ; u) X=\sum_{j \geq p+1} v_{j} Y_{j}(\xi)
$$


The dissipative inequality (8) thus writes

$$
V \cdot \sum_{j \geq p+1} v_{j} Y_{j}(\xi) \geq \omega(u) \sum_{\alpha}\left|\sum_{j \geq p+1} v_{j} Y_{j}^{\alpha}(\xi)\right|^{2}, \quad \forall V \in \mathbb{R}^{n}
$$

which amounts to

$$
\sum_{i, j \geq p+1} v_{i} v_{j} Y_{i j}(\xi) \geq \omega(u) \sum_{\alpha}\left|\sum_{j \geq p+1} v_{j} Z_{j}^{\alpha}(\xi)\right|^{2}, \quad \forall V \in \mathbb{R}^{n} .
$$

When $\xi \neq 0$, the right-hand side of $(14)$ is a norm for the vector $\left(v_{p+1}, \ldots, v_{n}\right)$, for if this quantity vanishes, then so does the linear combination

$$
\sum_{j \geq p+1} v_{j} Z_{j}(\xi)
$$

Since $\left\{Z_{p+1}(\xi), \ldots, Z_{n}(\xi)\right\}$ is a free family, this implies $v_{p+1}=\cdots=v_{n}=0$. Whence a positive number $c(\xi ; u)$ such that

$$
\sum_{i, j \geq p+1} v_{i} v_{j} Y_{i j}(\xi) \geq c(\xi ; u) \sum_{j \geq p+1} v_{j}^{2}
$$

By homogeneity of the left-hand side, we deduce that there exists a positive $c_{0}(u)$ such that

$$
\sum_{i, j \geq p+1} v_{i} v_{j} Y_{i j}(\xi) \geq c_{0}(u)|\xi|^{2} \sum_{j \geq p+1} v_{j}^{2}
$$

This is nothing but the Legendre-Hadamard condition for the four-indices tensor $Z_{i j}^{\alpha \beta}$.

To summarize, we have

Theorem 2.1 Assume that in an entropy-dissipative system (2), the p first rows are first-order conservation laws,

$$
\partial_{t} u_{j}+\operatorname{div} f_{j}(u)=0, \quad j=1, \ldots, p,
$$

while the symbol $B(\xi ; u)$ has rank $n-p$ for every non-zero $\xi \in \mathbb{R}^{d}$.

Then the second order part can be rewritten in the form

$$
\sum_{\alpha, \beta=1}^{d} \sum_{j=p+1}^{n} \partial_{\alpha}\left(Y_{j}^{\alpha \beta}(u) \partial_{\beta} \frac{\partial \eta}{\partial u_{j}}\right)
$$

with $Y^{\alpha \beta}=\left(\begin{array}{c}0 \\ Z^{\alpha \beta}\end{array}\right)$ satisfying the ellipticity property (Legendre-Hadamard condition)

$$
Z(\xi ; u) \geq c_{0}(u)|\xi|^{2} I_{n-p}, \quad\left(c_{0}(u)>0\right) .
$$




\section{Comments.}

1. This result tells us that the class of systems of the form

$$
\begin{aligned}
\partial_{t} v+\operatorname{div} g(v, w) & =0 \\
\partial_{t} w+\operatorname{div} h(v, w) & =\sum_{\alpha, \beta} \partial_{\alpha}\left(C^{\alpha \beta} \partial_{\beta} w\right),
\end{aligned}
$$

is not relevant from a physical point of view, unless the entropy $\eta$ splits as the sum of a $v$-part and a $w$-part ; in other words, unless $\mathrm{d}_{v} \mathrm{~d}_{w} \eta \equiv 0$. For an isentropic flow, the total energy plays the role of the entropy, and the property above applies in Lagrangian variables but it does not in Eulerian ones, a rather uncomfortable fact ! For a nonisentropic flow, the property applies only for an ideal gas (because then the temperature is a function of the internal specific energy $e$ only), and only in Lagrangian variables ; real gases or Eulerian variables cannot be handled within the class of systems $(15,16)$.

2. Let us assume that our system (2) is strongly entropy-dissipative. Then it is entropydissipative and therefore Theorem 2.1 applies. The tensor $Z$ however has the stronger ellipticity property that

$$
\sum_{\alpha, \beta=1}^{d} \sum_{i, j \geq p+1} F_{\alpha i} F_{\beta j} Z_{i j}^{\alpha \beta} \geq c_{0}(u)\|F\|^{2}, \quad \forall F \in \mathbf{M}_{d \times(n-p)}(\mathbb{R}) .
$$

In this situation, the dissipation rate takes the form

$$
\begin{aligned}
\sum_{\alpha, \beta} \mathrm{D}^{2} \eta(u)\left(\partial_{\alpha} u, B^{\alpha \beta}(u) \partial_{\beta} u\right) & =\sum_{\alpha, \beta=1}^{d} \sum_{i, j \geq p+1} Z_{i j}^{\alpha \beta} \partial_{\alpha} \frac{\partial \eta}{\partial u_{i}} \partial_{\beta} \frac{\partial \eta}{\partial u_{j}} \\
& \geq c_{0}(u)\left\|\nabla\left(\frac{\partial \eta}{\partial u_{p+1}}, \ldots, \frac{\partial \eta}{\partial u_{n}}\right)\right\|^{2}
\end{aligned}
$$

3. A similar structure occurs in systems of balance laws, called relaxation models. See for instance [15], where it is taken as a starting point.

\section{The reduced hyperbolic system}

We now consider the formal limit of the system

$$
\partial_{t} u+\sum_{\alpha} \partial_{\alpha} f^{\alpha}(u)=\kappa \sum_{\alpha, \beta} \partial_{\alpha}\left(B^{\alpha \beta}(u) \partial_{\beta} u\right)
$$

when $\kappa \rightarrow+\infty$. This limit is related to the time asymptotics $t \rightarrow+\infty$.

On the one hand, the system being dissipative, we have an estimate

$$
\int_{0}^{+\infty} \int_{\mathbb{R}}\left|B\left(u^{\kappa}\right) \nabla_{x} u^{\kappa}\right|^{2} d x d t \leq \frac{1}{\omega_{0} \kappa} \int_{\mathbb{R}} \eta\left(u_{0}^{\kappa}\right) d x
$$


where the right-hand side tends to zero if the initial data remains bounded. Denoting

$$
\mathbf{z}:=\left(\frac{\partial \eta}{\partial u_{p+1}}, \ldots, \frac{\partial \eta}{\partial u_{n}}\right)^{T}
$$

this amounts to

$$
\int_{0}^{+\infty} \int_{\mathbb{R}}\left|Z\left(u^{\kappa}\right) \nabla_{x} \mathbf{z}^{\kappa}\right|^{2} d x d t \leq \frac{1}{\omega \kappa} \int_{\mathbb{R}} \eta\left(u_{0}^{\kappa}\right) d x \stackrel{\kappa \rightarrow+\infty}{\longrightarrow} 0 .
$$

Thus it is likely, at least formally, that the limit $u$ is non-dissipated. Since the first order operator $Z(u) \nabla_{x}$ is elliptic, we expect that the limit $u$ takes values ${ }^{3}$ in a level set $\{u \mid \mathbf{z}=\mu\}$.

On the other hand, the $p$ first rows of the system do not depend on $\kappa$. Thus we expect that the limit $u$ satisfies them. Finally, our hope is that $v:=\left(u_{1}, \ldots, u_{p}\right)^{T}$ satisfies the closed system of first-order conservation laws

$$
\partial_{t} v+\sum_{\alpha} \partial_{\alpha} F^{\alpha}(v ; \mu)=0
$$

where $F$ is defined by $F_{j}^{\alpha}(v, \mathbf{z}):=f_{j}^{\alpha}(u)$ when $j=1, \ldots, p$. This definition turns out to be meaningful, because of the strong convexity of $\eta$ : the transformation

$$
u \mapsto(v, \mathbf{z})^{T}=\left(u_{1}, \ldots, u_{p}, \frac{\partial \eta}{\partial u_{p+1}}, \ldots, \frac{\partial \eta}{\partial u_{n}}\right)^{T}
$$

is a change of variable. Notice that if $u^{\prime} \neq u$, then

$$
\left\langle\mathrm{d} \eta\left(u^{\prime}\right)-\mathrm{d} \eta(u), u^{\prime}-u\right\rangle>0
$$

by strict convexity, and the left-hand side is linear in $(v, \mathbf{z})$. Therefore this strict inequality does not permit $(v, \mathbf{z})=\left(v^{\prime}, \mathbf{z}^{\prime}\right)$. In other words, the map $u \mapsto(v, \mathbf{z})$ is one-to-one.

The above analysis suggests the following

Definition 3.1 System (18) is called the reduced hyperbolic system of the viscous one (2).

This terminology anticipates on the following result, which is due to Boillat \& Ruggeri [1].

Theorem 3.1 Let the viscous system (2) be as above: entropy-dissipation, $B(\xi ; u)$ having a constant range as $\xi \neq 0$.

Then the reduced hyperbolic system admits a strongly convex entropy. In particular, it is Friedrichs symmetrizable, hence hyperbolic.

Proof

Let us denote

$$
\lambda_{j}:=\frac{\partial \eta}{\partial u_{j}}
$$

\footnotetext{
${ }^{3}$ Here, we go a little beyond the claim, by saying that in the limit, the variable $\mathbf{z}$ is also time-independent.
} 
the dual variables. In particular $\lambda_{j}=z_{j}$ for $j \geq p+1$. We shall write $\theta$ for $\left(\lambda_{1}, \ldots, \lambda_{p}\right)^{T}$. The Legendre-Fenchel transform $\eta^{*}$ of $\eta$ is a strongly convex function of $\lambda$, with

$$
\mathrm{D}_{\lambda}^{2} \eta^{*}=\left(\mathrm{D}_{u}^{2} \eta\right)^{-1}
$$

Recall (Godunov [6, 7], Friedrichs \& Lax [11]) that we have $u=\mathrm{d} \eta^{*}(\lambda)$ and that there exists smooth functions $M^{\alpha}(\lambda)$ such that $f^{\alpha}(u)=\mathrm{d} M^{\alpha}(\lambda)$ (actually, $M^{\alpha}(\lambda):=f^{\alpha}(u) \cdot \lambda-Q^{\alpha}(u)$, with $Q$ the entropy-flux). Since $\lambda=(\theta, \mathbf{z})$, the reduced hyperbolic system writes

$$
\partial_{t}\left(\mathrm{~d} \hat{\eta}^{*}(\theta)\right)+\sum_{\alpha} \partial_{\alpha}\left(\mathrm{d} \hat{M}^{\alpha}(\theta)\right)=0
$$

where $\hat{g}(\theta):=g(\theta, \mu)$. Since the restriction of $\eta^{*}$ to the linear subspace $\mathbf{z} \equiv \mu$ is a strongly convex function, we have our Friedrichs symmetrization:

$$
\mathrm{D}_{\theta}^{2} \hat{\eta}^{*} \partial_{t} \theta+\sum_{\alpha} \mathrm{D}_{\theta}^{2} \hat{M} \partial_{\alpha} \theta=0
$$

From (19), we also deduce an additional conservation law

$$
\partial_{t}\left(\mathrm{~d} \hat{\eta}^{*} \cdot \theta-\hat{\eta}^{*}\right)+\sum_{\alpha} \partial_{\alpha}\left(\mathrm{d} \hat{M}^{\alpha} \cdot \theta-\hat{M}^{\alpha}\right)=0
$$

With $v=\mathrm{d} \hat{\eta}^{*}$, the expression $E:=\mathrm{d} \hat{\eta}^{*} \cdot \theta-\hat{\eta^{*}}$ is nothing but the Legendre-Fenchel transform of $\hat{\eta}^{*}$, thus is a strongly convex function of $v$. This is the entropy of the reduced system.

\section{Practical issues.}

- From the proof above, we have

$$
E=\mathrm{d}_{\theta} \eta^{*} \cdot \theta-\eta^{*}=\mathrm{d}_{v} \eta \cdot v-\eta^{*},
$$

which yields the explicit formula

$$
E=\eta-\mathrm{d}_{w} \eta \cdot w
$$

where $w=\left(u_{p+1}, \ldots, u_{n}\right)$.

- Let us write blockwise the Hessian matrix of $\eta$ at $u=(v, w)^{T}$ :

$$
\mathrm{D}^{2} \eta(u)=\left(\begin{array}{cc}
s & r^{T} \\
r & \sigma
\end{array}\right)>0_{n},
$$

with $s \in \mathbf{S P D}_{p}$. Then the Hessian of the entropy of the reduced system at $v$, when $\mathrm{d}_{w} \eta=\mu$ is fixed, is given by the Schur complement $s-r^{T} \sigma^{-1} r$ of $\sigma$. It is a classical fact that since $\mathrm{D}^{2} \eta$ is positive definite, $s-r^{T} \sigma^{-1} r$ is so. 
The sub-characteristic property. In a hyperbolic first-order system $z_{t}+A(z) z_{x}=0$, infinitesimal disturbances, for instance singularities of derivatives, travel at finite speeds, the characteristic speeds. These are the eigenvalues $a_{-}:=a_{1} \leq \cdots \leq a_{n}=: a_{+}$of $A(z)$ and thus are functions of the state $z$. More generally, for a system written in the form $A_{0}(z) z_{t}+A(z) z_{x}=0$, the velocities are the generalized eigenvalues of the pair $\left(A_{0}, A\right)$, given by the equation

$$
\operatorname{det}\left(A(z)-a_{j}(z) A_{0}(z)\right)=0 .
$$

If the system is in symmetric form $\left(A_{0}(z)\right.$ and $A(z)$ symmetric, with $A_{0}(z)$ positive definite), the characteristic speeds may be written as Weyl's infsup formulæ of Rayleigh ratio. In particular

$$
a_{-}(z)=\inf _{X \neq 0} \frac{(A(z) X, X)}{\left(A_{0}(z) X, X\right)}, \quad a_{+}(z)=\sup _{X \neq 0} \frac{(A(z) X, X)}{\left(A_{0}(z) X, X\right)} .
$$

Let us apply this principle to the hyperbolic system (1)

$$
\partial_{t} u+\sum_{\alpha} \partial_{\alpha} f^{\alpha}(u)=0
$$

which has the symmetric form

$$
\mathrm{D}_{\lambda}^{2} \eta^{*} \partial_{t} \lambda+\sum_{\alpha} \mathrm{D}_{\lambda}^{2} M^{\alpha} \partial_{\alpha} \lambda=0
$$

and to the reduced system (18), in symmetric form (20). One passes from the former to the latter by replacing a symmetric pair $\left(A_{0}(u), A(\xi ; u)\right)$ by the pair $\left(S_{0}(u), S(\xi ; u)\right)$ of upper-left $p \times p$ blocs. In the infsup formulæ, this amounts to restrict to vectors $X$ of the form $(Y, 0)^{T}$ with $Y \in \mathbb{R}^{p}$. Making this restriction to increase infima and to lower suprema. We therefore obtain the following result, called sub-characteristic property.

Proposition 3.1 With the same assumptions as above, the characteritic velocities $a_{j}(\xi ; u)$ of (1) and those $b_{j}(\xi ; u)$ of the reduced system (18) satisfy the inequalities

$$
a_{j} \leq b_{j} \leq a_{j+n-p}, \quad j=1, \ldots, p .
$$

Conclusion. An entropy-dissipative viscous extension of a given hyperbolic system of conservation laws is far from being arbitrary, since the dissipation can concern only some very special quantities. As an example, we take again the gas dynamics. The entropy, in our mathematical sense, is $\eta=-\rho s$ ( $s$ the physical entropy), while the conserved variables are $u=\left(\rho, m=\rho v, \varepsilon:=\frac{1}{2} \rho v^{2}+\rho e\right)^{T}$. The above analysis tells us that $\partial \eta / \partial \varepsilon$ must be a function of $\theta$ only (since the Fourier-Euler system is dissipative), and that similarly $\partial \eta / \partial m$ must be a function of $(v, \theta)$ only (since the Fourier-Navier-Stokes system is dissipative too). We leave the reader establishing the identity

$$
\theta \mathrm{d} \eta=\left(e+\frac{p}{\rho}-\theta s-\frac{1}{2} v^{2}\right) \mathrm{d} \rho+v \mathrm{~d} m-\mathrm{d} \varepsilon,
$$


which confirms these assertions. Hint: start from $\theta \mathrm{d} s=\mathrm{d} e+p \mathrm{~d} \frac{1}{\rho}$.

If we start from the Fourier-Euler system $(n=2+d$ and $p=1+d)$, the reduced hyperbolic system is the isothermal Euler system. Proposition 3.1 tells that the sound speed in the adiabatic system (full Euler equations) dominates that in the isothermal system. We now calculate the reduced entropy $E$, constructed in the proof of Theorem 3.1. To do so, we employ Formula (21),

$$
\begin{aligned}
E & =\eta-\varepsilon \frac{\partial \eta}{\partial \varepsilon}=-\rho s+\frac{\varepsilon}{\theta} \\
& =\frac{1}{\theta}\left(\rho e-\rho \theta s+\frac{1}{2} \rho|v|^{2}\right) .
\end{aligned}
$$

We deduce that $E$ is nothing but the mechanical energy, renormalized by the temperature. The internal energy per unit mass is now

$$
e_{0}(\rho ; \theta):=e-\theta s
$$

also known as the free energy.

\section{References}

[1] G. Boillat, T. Ruggeri. Hyperbolic principal subsystems: Entropy convexity and subcharacteristic conditions. Arch. Rational Mech. Anal., 137 (1997), pp 305-320.

[2] C.M.Dafermos, Hyperbolic Conservation Laws in Continuum Physics. Second edition. Grundlehren der Mathematischen Wissenschaften 325, Springer Verlag, 2005.

[3] C. Dafermos, L. Hsiao. Global smooth thermomechanical processes in one-dimensional nonlinear thermoviscoelasticity. Nonlinear Anal. T.M.A., 6 (1982), pp 435-454.

[4] C. Dafermos, L. Hsiao. Development of singularities in solutions of the equations of nonlinear thermoelasticity. Quart. Appl. Math., XLIV (1986), pp 463-474.

[5] V. Giovangigli, M. Massot. Entropic structure of multicomponent reactive flows with partial equilibrium reduced chemistry. Math. Methods Appl. Sci., 27 (2004), pp 739-768.

[6] S. K. Godunov. An interesting class of quasi-linear systems. (Russian) Dokl. Akad. Nauk SSSR, 139 (1961), pp 521-523.

[7] S. K. Godunov. Lois de conservation et intégrales d'énergie des équations hyperboliques. Nonlinear Hyperbolic Problems (Carasso, Rascle \& Serre eds), St-Etienne 1986. Springer LNM 1270 (1987), pp 135-149.

[8] L. Hsiao, Quasilinear Hyperbolic Systems and Dissipative Mechanisms. World Scientific, 1997. 
[9] S. Kawashima. Systems of a hyperbolic parabolic type with applications to the equations of magnetohydrodynamics. PhD Thesis, Kyoto University (1983).

[10] S. Kawashima, Y. Shizuta. On the normal form of the symmetric hyperbolic-parabolic systems associated with the conservation laws. Tóhoku Math. J., 40 (1988), pp 449-464.

[11] K.-O. Friedrichs, P. D. Lax. Systems of conservation equations with a convex extension. Proc. Nat. Acad. Sci. U.S.A., 68 (1971), pp 1686-1688.

[12] T.-P. Liu, Y. Zeng. Large time behavior of solutions for general quasilinear hyperbolicparabolic systems of conservation laws. Mem. Amer. Math. Soc. 125 (1997), no. 599.

[13] D. Serre. Systems of conservation laws, Vol. I, Hyperbolicity, entropies, shock waves. Cambridge Univ. Press. Cambridge (1999).

[14] Y. Shizuta, S. Kawashima. Systems of equations of hyperbolic-parabolic type with applications to the discrete Boltzmann equation. Hokkaido Math. J., 14 (1985), pp 249-275.

[15] W.-A. Yong. An interesting class of partial differential equations. J. Math. Phys., 49 (2008), no. 3, 033503. In press. 